\title{
多酯基取代的叶绿素类二氢卟吩衍生物的合成
}

\author{
殷军港 ${ }^{a}$ 王 振 ${ }^{b}$ 杨 泽 ${ }^{a}$ 刘 超 ${ }^{b}$ \\ 赵丽丽 $a$ 王进军*,

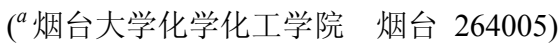 \\ ( 烟台大学药学院 烟台 264005)
}

\begin{abstract}
摘要 以脱镁叶绿酸-a 甲酯为起始原料, 通过碱性条件下的水解开环、空气氧化和重排反应, 分别合成了红紫素-7 三 甲酯和二氢卟吩- $\mathrm{p}_{6}$ 三甲酯. 然后对其 $\mathrm{C}(3)$-乙烯基、20-meso-位、12-位甲基以及尾端酯基进行化学修饰, 通过亲电加成、 亲电取代、1,3-偶极环加成和氧化重排等反应, 完成 10 种具未见报道的的叶绿素类二氢卟吩衍生物, 其化学结构均经 $\mathrm{UV}, \mathrm{IR},{ }^{1} \mathrm{H}$ NMR 光谱及元素分析予以表征.
\end{abstract}

关键词 叶绿素-a; 脱镁叶绿酸-a 甲酯; 叶绿素降解; 二氢卟吩; 化学修饰; 合成

\section{Synthesis of Multi-ester Substitutied Chlorin Derivatives Related to Chlorophyll-a}

\author{
Yin, Jungang $^{a} \quad$ Wang, Zhen ${ }^{b} \quad$ Yang, Ze $^{a} \quad$ Liu, Chao $^{b} \quad$ Zhao, Lili $^{a} \quad$ Wang, Jinjun ${ }^{*, a}$ \\ ( ${ }^{a}$ Colledge of Chemistry and Chemical Engineering, Yantai University, Yantai 264005) \\ ( ${ }^{b}$ Pharmacy College, Yantai University, Yantai 264005)
}

\begin{abstract}
From methyl pheophorbide-a which was used as a starting material, purpurin-7 trimethyl ester and chlorin- $\mathrm{p}_{6}$ trimethyl ester were synthesized by hydrogation-opening ring, allomerization and rearrangement reaction. The synthesis of ten unreported chlorin derivatives related to chlorophyll-a was accomplished by electrophilic adduction, electrophilic substitution, 1,3-dipolar cycloadduction and oxidation-rearrangement reaction to chemical modifications for $\mathrm{C}(3)$-vinyl group, 20-meso-position, methyl group at 12-position and ester moieties at terminal positions. The structures of all new chlorins were characterized by UV, IR, ${ }^{1} \mathrm{H}$ NMR spectra and elemental analysis.
\end{abstract}

Keywords chlorophyll-a; methyl pheophorbide-a; chlorophyll degradation; chlorin; chemical modification; synthesis

叶绿素-a 及其降解产物的二氢卟吩共轭大环中的 非对称性化学结构和多取代活性官能团, 是合成高效光 动力治疗 (phtodynamic therapy, 简称 PDT) 抗癌药物的 基本条件. 特别是许多叶绿素- $\mathrm{a}$ 的降解产物在 C-D 环端 向含有 $\beta$-酮酯、 $\alpha$-酮酯、芳香和脂肪羧酸酯结构, 这些 吸电子性的多氧基团与 A-B 环上的烃基一起赋予了二 氢卟吩大环分子良好的水脂溶性, 使得 PDT 过程中所 必需的生物利用度得到保证. 同时, 对于叶绿素衍生物 多酯结构的化学修饰和结构改造也是合成新型二氢卟 吩的重要方法 ${ }^{[1 \sim 3]}$. 二氢卟吩- $\mathrm{e}_{6}$ 三甲酯、红紫素-7 三甲 酯和二氢卟吩- $\mathrm{p}_{6}$ 三甲酯在 C-D 环端向分别连有三个酯
基，其多酯羰基的转换和重排可以巧妙地构建出各种不 同形式的新颖结构, 也相应地形成了许多具有新意的理 论解释, 从不同层面上丰富和拓宽了叶绿素化学的理论 和应用研究 ${ }^{[4 \sim 6]}$. 本文基于前期的研究工作 ${ }^{[7,8]}$, 通过对 上述多酯基叶绿素衍生物实施化学修饰, 在大环分子上 构建和引进不同的化学结构和取代基团, 完成一系列未 见报道的多酯基叶绿素类二氢卟吩衍生物的合成.

以脱镁叶绿酸-a 甲酯 $(1, M P-a)$ 为起始原料, 在氢氧 化钾存在下经历了开环和空气氧化反应, MP-a (1)转换 成红紫素-7 三甲酯 2 (72\%). 在含有氢氧化钾的丙酮水 溶液中, 起始原料回流 $2 \mathrm{~h}$ 后高产率地转化成红紫素-18

*E-mail: wjj1955@153.com

Received August 30, 2011; revised October 9, 2011; accepted October 25, 2011.

Project supported by the Natural Science Foundation of Shandong Province of China (No. Y2008B49) and the International Science and Technology Project between Governments of China and Hungary (No. 2008-444-4-32).

山东省自然科学基(No. Y2008B49)、中匈政府间科技合作(No. 2008-444-4-32)资助项目. 


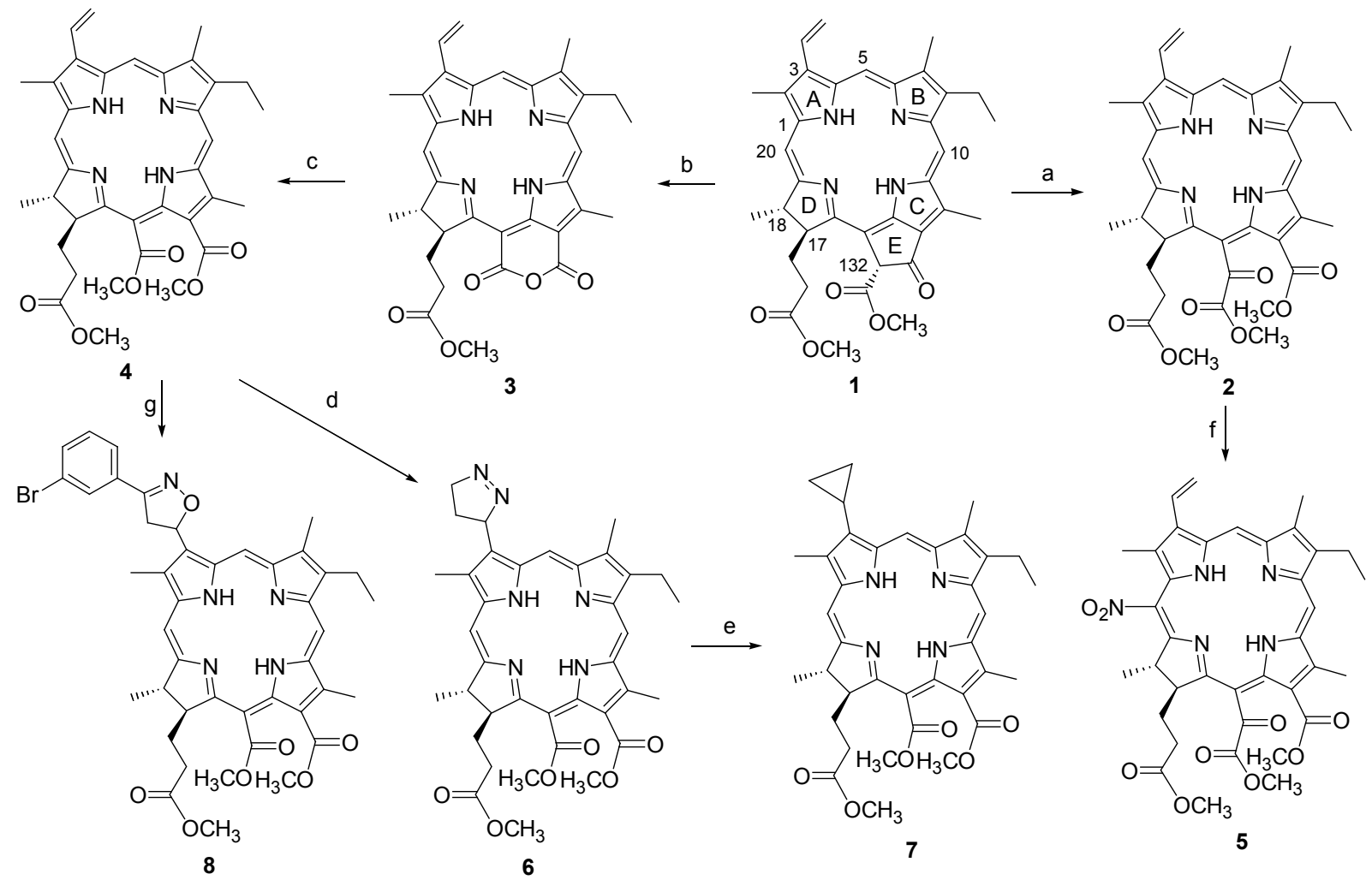

Reagents and Conditions: (a) $\mathrm{KOH} / \mathrm{O}_{2} / \mathrm{C}_{3} \mathrm{H}_{7} \mathrm{OH} / \mathrm{CH}_{2} \mathrm{~N}_{2}$; (b) $\mathrm{KOH}, \mathrm{O}_{2} / \mathrm{CO}\left(\mathrm{CH}_{3}\right)_{2} / \mathrm{CH}_{2} \mathrm{~N}_{2}$; (c) $\mathrm{KOH} / \mathrm{MeOH} / \mathrm{CH}_{2} \mathrm{~N}_{2}, 0{ }^{\circ} \mathrm{C}$; (d) $\mathrm{CH}_{2} \mathrm{~N}_{2} / \mathrm{CH}_{2} \mathrm{Cl}_{2}$; (e) $\mathrm{PhOPh} / 18_{0}{ }^{\circ} \mathrm{C}$; (f) $\mathrm{HNO}_{3} / \mathrm{AcOH}$; (g) $\mathrm{ArCNOH} / \mathrm{NBS} / \mathrm{N}(\mathrm{Et})_{3}$

\section{Scheme 1}

甲酯 3. 在低温条件下经碱处理开环, 酸化后再以重氮 甲烷甲基化, 则以 $77 \%$ 的产率得到二氢卟吩 $-\mathrm{p}_{6}$ 三甲酯 4. 利用浓硝酸对化合物 2 实施硝化, 则以 $63 \%$ 的产率给 出 20-位硝基取代的红紫素-7 三甲酯 $\mathbf{5}$. 化合物 4 与重氮 甲烷可以顺利发生 1,3-偶极环加成反应，得到 $60 \%$ 的 $\mathrm{C}(3)$-吡唑啉基取代的二氢卟吩- $\mathrm{p}_{6}$ 三甲酯 6 . 然后, 将所 得加成物于 $180{ }^{\circ} \mathrm{C}$ 的二苯醚中搅拌反应, 几乎定量地 裂解为环丙基二氢卟吩 7. 在三乙胺存在下, 选用 $N$-溴 代丁二酰亚胺(NBS)对苯甲醛肜实施溴代和碱催化脱卤 化氢, 所生成的氧化苯甲腈不经分离直接与二氢卟吩$\mathrm{p}_{6} 4$ 进行 1,3-偶环加成反应, 以 70\%的产率分离出 $\mathrm{C}(3)-$ 间溴苯基异噁唑取代的 1,3-偶极环加成产物 8 .

考虑到化合物 2 中 15-位上的 $\alpha$-酩酯应该具有更加 活泼的反应活性，选用甲基三苯基鏻与其进行 Wittig 反 应, 结果低产率的分离出二氢卟吩三酯 $(9,11 \%)$; 尝试 用硼氢化钠还原红紫素-7 三甲酯 2 的 15-位酮基, 以期 将其转化成二氢卟吩 $-\mathrm{e}_{6}$ 三甲酯. 结果从复杂的混合物 中只分离出 $21 \%$ 的红紫素-7 内酯 10. 为了拓宽 C(3)-位 的乙烯基在化学修饰中的应用范围, 用溴化氢乙酸溶液 处理化合物 2, 试图得到具有高反应活性的溴代二氢卟 吩, 然而却分离出 54\%的溴代水解产物 11. 参考具有五 元外接环结构的焦脱镁叶绿酸的空气氧化反应 ${ }^{[9,10]}$, 在
相同的碱性条件下对二氢卟吩- $\mathrm{p}_{6} 2$ 实施空气氧化，分别 低产率地得到二氢卟吩醛 12 (11\%)和 12-甲酰基红紫卟 啉 $13(3 \%)$. 在多酸共存条件下，化合物 2 与 $40 \%$ 的甲醛 溶液发生羟醛缩合反应, 结果低产率地分离出 12-羟乙 基取代的二氢卟吩- $\mathrm{p}_{6} 14$ (Scheme 2).

\section{1 实验部分}

元素分析用 Perkin-Elmer 2400 型元素分析仪测定; IR 用 Perkin-Elmer 1730 型红外分光光度仪测定 $(\mathrm{KBr}$ 压 片); UV-vis 用 UV-160A 型紫外分光光度计测定; ${ }^{1} \mathrm{H}$ NMR 用 Brucker ARX-300 型核磁共振仪测定, 内标为 TMS. 所用试剂均为分析纯和化学纯. 脱镁叶绿酸-a 甲 酯 1 按文献[11]制备; 红紫素-7 三甲酯 2、红紫素-18 甲 酯 3 和二氢卟吩- $p_{6}$ 三甲酯 4 按文献[12]制备;

\subsection{0 -硝基红紫素-7 三甲酯(5)的合成}

将 $212 \mathrm{mg}$ (0.349 mmol) 1 溶解于 $50 \mathrm{~mL}$ 二氯甲烷 中, 冷却至 $0{ }^{\circ} \mathrm{C}$, 剧烈搅拌下滴加溶解于 $5 \mathrm{~mL}$ 乙酸的 $0.2 \mathrm{~mL}$ 浓硝酸, 滴加完毕后在相同温度下搅拌反应 $2 \mathrm{~h}$, 然后加入 $50 \mathrm{~mL}$ 水, 用 $5 \%$ 的碳酸氢钠溶液洗涤有机层, 无水硫酸钠干燥后减压除去溶剂, 通过柱层析分离混合 物[洗脱剂: $V$ (乙酸乙酯) $: V($ 正己烷 $)=1: 3$ ]得 $154 \mathrm{mg}$ 


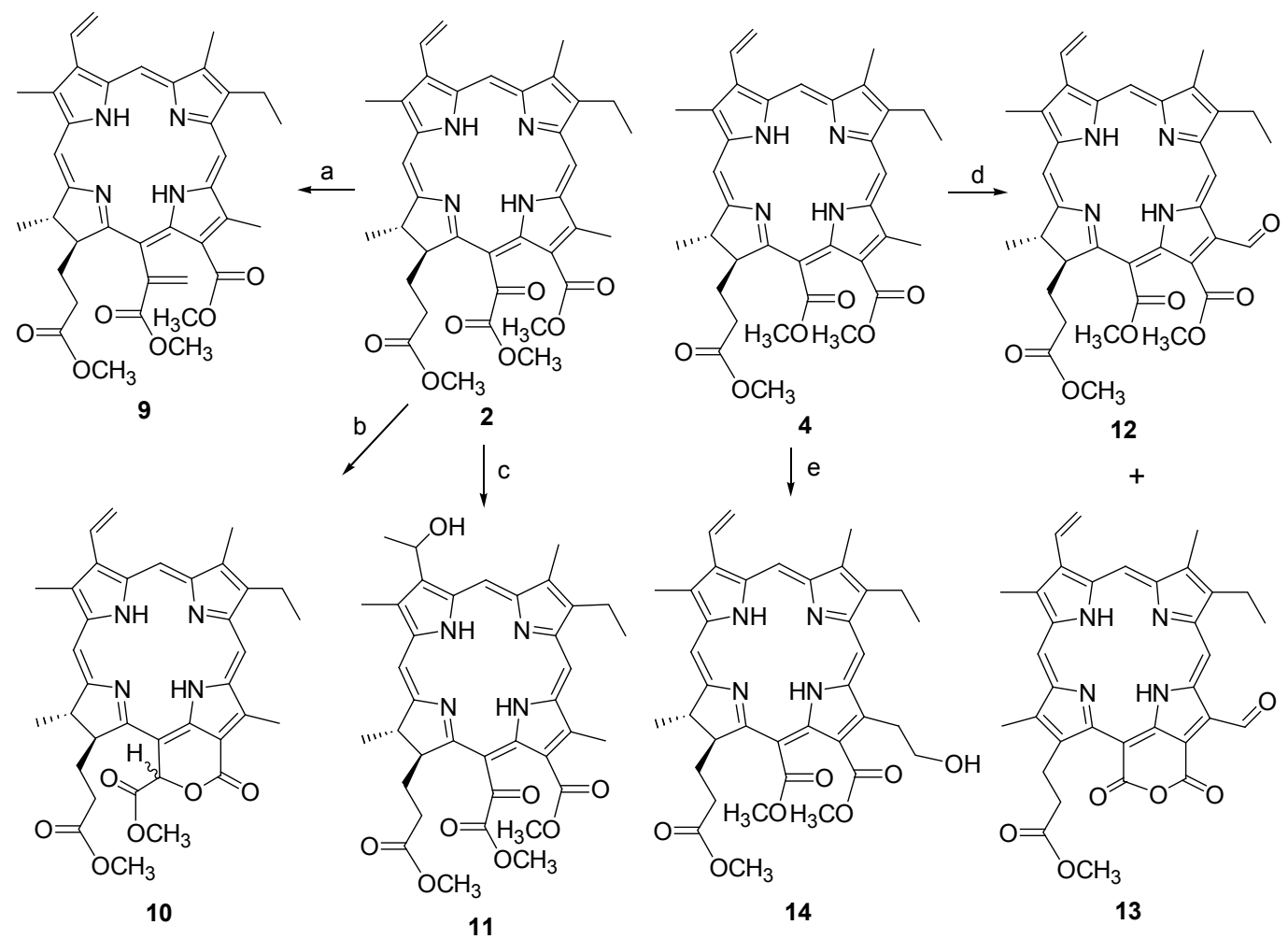

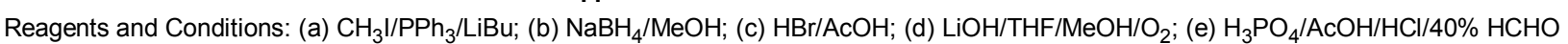

Scheme 2

(0.220 mmol)红色固体 5. 产率为 63\%. m.p. $215 \sim 217$ ${ }^{\circ} \mathrm{C}$; UV-vis $\left(\mathrm{CHCl}_{3}\right) \lambda_{\max }\left[\varepsilon /\left(\mathrm{L} \cdot \mathrm{mol}^{-1} \cdot \mathrm{cm}^{-1}\right)\right]: 407$ (120000), 507 (8400), 546 (14400), 677 (31200) nm; ${ }^{1} \mathrm{H}$ $\operatorname{NMR}\left(\mathrm{CDCl}_{3}\right) \delta:-2.32($ br s, $1 \mathrm{H}, \mathrm{NH}),-2.48($ br s, $1 \mathrm{H}$, $\mathrm{NH}), 1.69$ (t, $\left.J=7.6 \mathrm{~Hz}, 3 \mathrm{H}, 8-\mathrm{CH}_{3}\right), 1.70(\mathrm{~d}, J=7.3 \mathrm{~Hz}$, $\left.3 \mathrm{H}, 18-\mathrm{CH}_{3}\right), 2.14 \sim 2.32(\mathrm{~m}, 2 \mathrm{H}, 17 \mathrm{a}+17 \mathrm{~b}-\mathrm{H}), 2.52 \sim$ $2.61(\mathrm{~m}, 2 \mathrm{H}, 17 \mathrm{a}+17 \mathrm{~b}-\mathrm{H}), 3.76(\mathrm{q}, J=7.6 \mathrm{~Hz}, 2 \mathrm{H}, 8 \mathrm{a}-\mathrm{H})$, 3.37 (s, $3 \mathrm{H}, \mathrm{CH}_{3}+\mathrm{OCH}_{3}$ ), 3.44 (s, $\left.3 \mathrm{H}, \mathrm{CH}_{3}+\mathrm{OCH}_{3}\right), 3.56$ (s, $\left.3 \mathrm{H}, \mathrm{CH}_{3}+\mathrm{OCH}_{3}\right), 3.62\left(\mathrm{~s}, 3 \mathrm{H}, \mathrm{CH}_{3}+\mathrm{OCH}_{3}\right), 3.81$ (s, $\left.3 \mathrm{H}, \mathrm{CH}_{3}+\mathrm{OCH}_{3}\right), 4.82\left(\mathrm{~s}, 3 \mathrm{H}, \mathrm{CH}_{3}+\mathrm{OCH}_{3}\right), 4.39$ (d, $J=$ $8.6 \mathrm{~Hz}, 1 \mathrm{H}, 17-\mathrm{H}), 4.45$ (q, $J=7.3 \mathrm{~Hz}, 1 \mathrm{H}, 18-\mathrm{H}), 5.23$ (d, $J=18.9 \mathrm{~Hz}, 1 \mathrm{H}, 15 \mathrm{a}-\mathrm{H}), 5.35(\mathrm{~d}, J=18.9,1 \mathrm{H}, 15 \mathrm{a}-\mathrm{H})$, 6.11 (dd, $J=11.6,1.2 \mathrm{~Hz}, 1 \mathrm{H}$, cis-3b-H), 6.32 (dd, $J=$ $17.8,1.2 \mathrm{~Hz}, 1 \mathrm{H}$, trans-3b-H), $8.02(\mathrm{dd}, J=17.8,11.6 \mathrm{~Hz}$, 1H, 3a-H), 9.47 (s, 1H, meso-H), 9.50 (s, 1H, meso-H); IR (KBr) v: $3444(\mathrm{~N}-\mathrm{H}), 1972,2935(\mathrm{C}-\mathrm{H}), 1741-1681$ $(\mathrm{C}=\mathrm{O}), 1687,1618(\mathrm{C}=\mathrm{C}), 1562$ (chlorin skeleton), 1456, 1373, 1176, 1014, $958 \mathrm{~cm}^{-1}$. Anal. calcd for $\mathrm{C}_{37} \mathrm{H}_{39^{-}}$ $\mathrm{N}_{5} \mathrm{O}_{9}$ : C 63.69, H 5.63, N 10.04; found C 63.46, H 5.78, N 10.17 .

1.23 -[3'(R,S)-(1'-吡唑啉基)]-3-去乙烯基卟吩- $\mathrm{p}_{6}$ 三甲 酯(6)的合成

$88 \mathrm{mg} 4(0.141 \mathrm{mmol})$ 溶解于 $3 \mathrm{~mL}$ 二氯甲烷中, 加
入 $25 \mathrm{~mL}$ 重氮甲烷乙醚溶液，室温下避光密闭放置 $32 \mathrm{~h}$, 搅拌下加入 $5 \mathrm{~mL}$ 乙酸以除去多余的重氮甲烷, 然后加 入 $30 \mathrm{~mL}$ 二氯甲烷和 $30 \mathrm{~mL}$ 水分层, 有机相用水洗涤三 次，分出有机层，用无水硫酸钠干燥，减压浓缩，所得 剩余物用硅胶柱层析分离[洗脱剂: $V$ (乙酸乙酯)： $V$ (石 油醚 $)=1: 3]$, 得到 $57 \mathrm{mg}(0.085 \mathrm{mmol})$ 暗红色固体 $\mathbf{6}$, 产率 60\%. m.p. 225 228 ${ }^{\circ} \mathrm{C}$; UV-vis $\left(\mathrm{CHCl}_{3}\right) \lambda_{\max }$

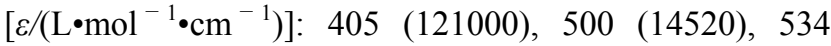
(6050), 606 (7240), 661 (45980) nm; ${ }^{1} \mathrm{H}$ NMR $\left(\mathrm{CDCl}_{3}\right) \delta$ : -1.03 (br s, $1 \mathrm{H}, \mathrm{NH}), 0.33$ (br s, $1 \mathrm{H}, \mathrm{NH}), 1.68$ (t, $J=7.6$ $\left.\mathrm{Hz}, 3 \mathrm{H}, 8-\mathrm{CH}_{3}\right), 1.82\left(\mathrm{~d}, J=7.4 \mathrm{~Hz}, 3 \mathrm{H}, 18-\mathrm{CH}_{3}\right), 2.00 \sim$ $2.82\left(\mathrm{~m}, 6 \mathrm{H}, 4^{\prime}-\mathrm{H}+17 \mathrm{a}+17 \mathrm{~b}-\mathrm{H}\right), 3.72(\mathrm{q}, J=7.6 \mathrm{~Hz}, 2 \mathrm{H}$, $8 \mathrm{a}-\mathrm{H}), 3.18$ (s, $\left.3 \mathrm{H}, \mathrm{CH}_{3}+\mathrm{OCH}_{3}\right), 3.27(3.26)(\mathrm{s}, 3 \mathrm{H}, \mathrm{CH}+$ $\left.\mathrm{OCH}_{3}\right), 3.52$ (s, $\left.3 \mathrm{H}, \mathrm{CH}_{3}+\mathrm{OCH}_{3}\right), 3.91\left(\mathrm{~s}, 3 \mathrm{H}, \mathrm{CH}_{3}+\right.$ $\left.\mathrm{OCH}_{3}\right), 4.17\left(\mathrm{~s}, 3 \mathrm{H}, \mathrm{CH}_{3}+\mathrm{OCH}_{3}\right), 4.23\left(\mathrm{~s}, 3 \mathrm{H}, \mathrm{CH}_{3}+\right.$ $\left.\mathrm{OCH}_{3}\right), 4.42$ (q, $\left.J=7.0 \mathrm{~Hz}, 1 \mathrm{H}, 18-\mathrm{H}\right), 5.17(\mathrm{~d}, J=8.6 \mathrm{~Hz}$, $1 \mathrm{H}, 17-\mathrm{H}), 4.67 \sim 4.82\left(\mathrm{~m}, 1 \mathrm{H}, 5^{\prime}-\mathrm{H}\right), 5.39 \sim 5.50(\mathrm{~m}, 1 \mathrm{H}$, 5'-H), 6.68 (t, $\left.J=8.6 \mathrm{~Hz}, 1 \mathrm{H}, 3^{\prime}-\mathrm{H}\right), 8.70$ (s, 1H, meso-H), $9.11(\mathrm{~s}, 1 \mathrm{H}$, meso-H), $9.71(\mathrm{~s}, 1 \mathrm{H}$, meso-H); IR $(\mathrm{KBr}) v$ : $3458(\mathrm{~N}-\mathrm{H}), 2968,2933(\mathrm{~N}-\mathrm{H}), 1743 \sim 1706(\mathrm{C}=\mathrm{O})$, $1608(\mathrm{C}=\mathrm{C}), 1548$ (chlorin skeleton), 1409, 1255, 1708, $908,853 \mathrm{~cm}^{-1}$. Anal. calcd for $\mathrm{C}_{37} \mathrm{H}_{42} \mathrm{~N}_{6} \mathrm{O}_{6}: \mathrm{C} 66.65, \mathrm{H}$ 6.35, N 12.60; found C 66.46, H 6.48, N 12.77 . 


\subsection{3-环丙基-3-去乙烯基卟吩- $\mathrm{p}_{6}$ 三甲酯(7)的合成}

$55 \mathrm{mg}$ 化合物 $6(0.083 \mathrm{mmol})$ 溶于 $25 \mathrm{~mL}$ 二苯醚中, 于 $180{ }^{\circ} \mathrm{C}$ 油浴摚拌反应 $20 \mathrm{~min}$, 加入正己烷稀释, 过滤 除去二苯醚, 以少量二氯甲烷溶解固体混合物, 硅胶柱 层析分离 [洗脱剂: $V$ (乙酸乙酯) : $V$ (石油醚) $=1: 3$ ], 得 $48 \mathrm{mg}$ (0.075 mmol) 墨绿色固体 7, 产率 90\%. m.p. 199

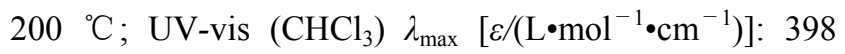
(118000), 496 (10620), 527 (0.04), 605 (4720), 658 (35400) nm; ${ }^{1} \mathrm{H} \mathrm{NMR}\left(\mathrm{CDCl}_{3}\right) \delta: 0.56$ (br s, $\left.1 \mathrm{H}, \mathrm{NH}\right),-$ 0.91 (br s, $1 \mathrm{H}, \mathrm{NH}), 1.30 \sim 1.38(\mathrm{~m}, 2 \mathrm{H}, 3 \mathrm{~b}-\mathrm{H}), 1.43 \sim 1.55$ (m, 2H, 3b-H), 1.68 (t, J=7.5 Hz, 3H, 8- $\left.\mathrm{CH}_{3}\right), 1.84$ (d, $\left.J=7.1 \mathrm{~Hz}, 3 \mathrm{H}, 18-\mathrm{CH}_{3}\right), 1.98 \sim 2.08(\mathrm{~m}, 1 \mathrm{H}, 17 \mathrm{a}+$ $17 \mathrm{~b}-\mathrm{H}), 2.16 \sim 2.24(\mathrm{~m}, 1 \mathrm{H}, 17 \mathrm{a}+17 \mathrm{~b}-\mathrm{H}), 2.30 \sim 2.42(\mathrm{~m}$, $2 \mathrm{H}, 17 \mathrm{a}+17 \mathrm{~b}-\mathrm{H}), 2.70 \sim 2.78(\mathrm{~m}, 1 \mathrm{H}, 3 \mathrm{a}-\mathrm{H}), 3.72$ (q, $J=$ $7.6 \mathrm{~Hz}, 2 \mathrm{H}, 8 \mathrm{a}-\mathrm{H}), 3.26\left(\mathrm{~s}, 3 \mathrm{H}, \mathrm{CH}_{3}+\mathrm{OCH}_{3}\right), 3.31(\mathrm{~s}, 3 \mathrm{H}$, $\left.\mathrm{CH}_{3}+\mathrm{OCH}_{3}\right), 3.51\left(\mathrm{~s}, 3 \mathrm{H}, \mathrm{CH}_{3}+\mathrm{OCH}_{3}\right), 3.62(\mathrm{~s}, 3 \mathrm{H}$, $\left.\mathrm{CH}_{3}+\mathrm{OCH}_{3}\right), 4.16\left(\mathrm{~s}, 3 \mathrm{H}, \mathrm{CH}_{3}+\mathrm{OCH}_{3}\right), 4.21(\mathrm{~s}, 3 \mathrm{H}$, $\left.\mathrm{CH}_{3}+\mathrm{OCH}_{3}\right), 4.36$ (q, $\left.J=7.0 \mathrm{~Hz}, 1 \mathrm{H}, 18-\mathrm{H}\right), 5.16$ (d, $J=$ $8.6 \mathrm{~Hz}, 1 \mathrm{H}, 17-\mathrm{H}), 8.54(\mathrm{~s}, 1 \mathrm{H}$, meso-H), $9.55(\mathrm{~s}, 1 \mathrm{H}$, meso-H), 9.64 (s, 1H, meso-H); IR (KBr) v: $3444(\mathrm{~N}-\mathrm{H})$, 2966, $2935(\mathrm{C}-\mathrm{H}), 1739 \sim 1695(\mathrm{C}=\mathrm{O}), 1654(\mathrm{C}=\mathrm{C})$, 1566 (chlorin skeleton), 1461, 1384, 1024, $742 \mathrm{~cm}^{-1}$. Anal. calcd for $\mathrm{C}_{37} \mathrm{H}_{42} \mathrm{~N}_{4} \mathrm{O}_{6}$ : C 69.57, H 6.63, N 8.77; found C 69.40, H 6.68, N 8.51.

\subsection{3-[5'(R,S)-(3'-间溴苯基-4',5'-二氢异噁唑基)]-3-去 乙烯基红紫素-7 三甲酯(8)的合成}

在 $1 \mathrm{~mL}$ 干燥二氯甲烷中, 加入 $98 \mathrm{mg}(0.562 \mathrm{mmol})$ $\mathrm{NBS}$, 一次性加入 $70 \mathrm{mg}(0.582 \mathrm{mmol})$ 苯甲醛肜, 溴化 $20 \mathrm{~min}$ 后结束反应. 然后加入 $361 \mathrm{mg}(0.578 \mathrm{mmol})$ 二氢 卟吩 $-\mathrm{p}_{6} 4$, 待其完全溶解后, 再将溶解在 $1 \mathrm{~mL}$ 二氯甲烷 中的 $70.4 \mathrm{mg}(0.704 \mathrm{mmol})$ 三乙胺在 $15 \mathrm{~min}$ 内滴入反应 液, 氮气保护, 室温搅拌反应 $24 \mathrm{~h}$, 加入二氯甲烷和水 萃取, 有机相水洗, 干燥后减压浓缩, 用 500 800 目硅 胶制备薄层色谱分离 [洗脱剂: $V($ 苯 $): V($ 丙酮 $)=10 ： 1$ ], 分别得到 $333 \mathrm{mg}$ 绿色固体 8 (0.405 mmol), 产率 70\%. m.p. $241 \sim 244{ }^{\circ} \mathrm{C}$; UV-vis $\left(\mathrm{CHCl}_{3}\right) \lambda_{\max }\left[\varepsilon /\left(\mathrm{L} \cdot \mathrm{mol}^{-1} \bullet\right.\right.$ $\left.\mathrm{cm}^{-1}\right)$ ]: 398 (119000), 498 (9520), 527 (5950), 605 (5950), 667 (48790) nm; ${ }^{1} \mathrm{H}$ NMR (400 MHz, $\left.\mathrm{CDCl}_{3}\right) \delta:-0.91$ (br s, $1 \mathrm{H}, \mathrm{NH}),-1.16$ (br s, $1 \mathrm{H}, \mathrm{NH}), 1.63(\mathrm{t}, J=7.6 \mathrm{~Hz}$, $\left.3 \mathrm{H}, 8-\mathrm{CH}_{3}\right), 1.87\left(\mathrm{~d}, J=6.8 \mathrm{~Hz}, 3 \mathrm{H}, 18-\mathrm{CH}_{3}\right), 2.03 \sim 2.12$ (m, 1H, 17a+17b-H), $2.17 \sim 2.28(\mathrm{~m}, 1 \mathrm{H}, 17 \mathrm{a}+17 \mathrm{~b}-\mathrm{H})$, $2.35 \sim 2.46(\mathrm{~m}, 2 \mathrm{H}, 17 \mathrm{a}+17 \mathrm{~b}-\mathrm{H}), 3.06\left(\mathrm{~s}, 3 \mathrm{H}, \mathrm{CH}_{3}+\right.$ $\left.\mathrm{OCH}_{3}\right), 3.42\left(\mathrm{~s}, 3 \mathrm{H}, \mathrm{CH}_{3}+\mathrm{OCH}_{3}\right), 3.52\left(\mathrm{~s}, 3 \mathrm{H}, \mathrm{CH}_{3}+\right.$ $\left.\mathrm{OCH}_{3}\right), 3.64\left(\mathrm{~s}, 3 \mathrm{H}, \mathrm{CH}_{3}+\mathrm{OCH}_{3}\right), 4.18\left(\mathrm{~s}, 3 \mathrm{H}, \mathrm{CH}_{3}+\right.$ $\left.\mathrm{OCH}_{3}\right), 4.23\left(\mathrm{~s}, 3 \mathrm{H}, \mathrm{CH}_{3}+\mathrm{OCH}_{3}\right), 3.63$ (q, $J=7.6 \mathrm{~Hz}, 2 \mathrm{H}$,
$8 \mathrm{a}-\mathrm{H}), 4.19$ (dd, $\left.J=18.0,10.7 \mathrm{~Hz}, 1 \mathrm{H}, 4^{\prime}-\mathrm{H}\right), 4.43$ (q, $J=$ $7.4 \mathrm{~Hz}, 18-\mathrm{H}$ ), 4.46 (dd, $\left.J=18.0,10.7 \mathrm{~Hz}, 1 \mathrm{H}, 4^{\prime}-\mathrm{H}\right), 5.19$ (d, $J=7.8 \mathrm{~Hz}, 17-\mathrm{H}), 7.16$ (t, $J=10.7 \mathrm{~Hz}, 5 '-\mathrm{H}), 7.33$ (7.32) (d, $J=7.7 \mathrm{~Hz}, 1 \mathrm{H}, \mathrm{Ph}-\mathrm{H}), 7.84$ (7.83) (dt, $J=7.7$, $3.2 \mathrm{~Hz}, 1 \mathrm{H}, \mathrm{Ph}-\mathrm{H}), 8.34$ (8.32) (d, $J=7.7 \mathrm{~Hz}, 1 \mathrm{H}, \mathrm{Ph}-\mathrm{H})$, 8.62 (8.61) (s, 1H, Ph-H), 8.70 (s, 1H, meso-H), 9.48 (s, $1 \mathrm{H}$, meso-H), 10.18 (s, 1H, meso-H); IR (KBr) v: 3439 $(\mathrm{N}-\mathrm{H}), 2972 \sim 2888(\mathrm{C}-\mathrm{H}), 1713 \sim 1728(\mathrm{C}=\mathrm{O}), 1618$ $(\mathrm{C}=\mathrm{C}), 1548$ (chlorin skeleton), 1521, 1410, 1071876 $\mathrm{cm}^{-1}$. Anal. calcd for $\mathrm{C}_{43} \mathrm{H}_{44} \mathrm{BrN}_{5} \mathrm{O}_{7}$ : C 62.77, H 5.39, N 9.71; found C 62.54, H 5.53, N 9.90.

\section{$1.515^{2}$-亚甲基-15 2 -去氧红紫素-7 三甲酯(9)的合成}

在带有回流冷凝管和滴液漏斗 $25 \mathrm{~mL}$ 三口瓶中加 入 $15 \mathrm{~mL}$ 干燥的四氢呋喃, 然后加入 $100 \mathrm{mg}$ 溴化甲基 三苯鏻细粉, 氮气保护条件下滴加 $1 \mathrm{~mL}$ 丁基锂正己烷 溶液 $(2.2 \mathrm{~mol} / \mathrm{L})$, 当固体完全溶解后再搅拌 $5 \mathrm{~min}$. 然后, 搅拌下滴入溶解于 $25 \mathrm{~mL}$ 无水四氢呋喃的 $165 \mathrm{mg}$ (0.253 mmol) 2 , 继续搅拌反应 $4 \mathrm{~h}$ 后, 缓慢加入 $35 \mathrm{~mL}$ 水和 $35 \mathrm{~mL}$ 二氯甲烷, 过滤, 分出有机层, 水层用氯仿 萃取 $(25 \mathrm{~mL} \times 2)$, 合并有机层, 冷水洗涤后以无水硫酸 钠干燥. 再用重氮甲烷处理有机相, 减压除去溶剂, 将 剩余物通过柱层析分离混合物 [洗脱剂: $V($ 乙酸乙 酯)： $V$ (正己 烷) $=1 ： 4]$ 得 $18 \mathrm{mg}(0.028 \mathrm{mmol})$ 红色固 体 9, 产率 9\%. m.p. 202 205 ${ }^{\circ} \mathrm{C}$; UV-vis $\left(\mathrm{CHCl}_{3}\right) \lambda_{\max }$

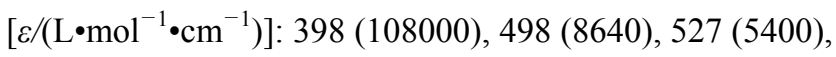
605 (5400), 667 (44280) nm; ${ }^{1} \mathrm{H}$ NMR (400 MHz. $\mathrm{CDCl}_{3}$ ) $\delta:-1.60($ br s, $1 \mathrm{H}, \mathrm{NH}),-1.77($ br s, $1 \mathrm{H}, \mathrm{NH}), 1.72(\mathrm{t}$, $\left.J=7.6 \mathrm{~Hz}, 3 \mathrm{H}, 8-\mathrm{CH}_{3}\right), 1.81\left(\mathrm{~d}, J=6.9 \mathrm{~Hz}, 3 \mathrm{H}, 18-\mathrm{CH}_{3}\right)$, $1.77 \sim 1.90(\mathrm{~m}, 1 \mathrm{H}, 17 \mathrm{a}+17 \mathrm{~b}-\mathrm{H}), 2.00 \sim 2.14(\mathrm{~m}, 2 \mathrm{H}$, $17 \mathrm{a}+17 \mathrm{~b}-\mathrm{H}), 2.20 \sim 2.35(\mathrm{~m}, 1 \mathrm{H}, 17 \mathrm{a}+17 \mathrm{~b}-\mathrm{H}), 3.80$ (q, $J=7.6 \mathrm{~Hz}, 2 \mathrm{H}, 8 \mathrm{a}-\mathrm{H}), 3.33\left(\mathrm{~s}, 3 \mathrm{H}, \mathrm{CH}_{3}+\mathrm{OCH}_{3}\right), 3.40$ (s, $\left.3 \mathrm{H}, \mathrm{CH}_{3}+\mathrm{OCH}_{3}\right), 3.49\left(\mathrm{~s}, \mathrm{CH}_{3}+\mathrm{OCH}_{3}\right), 3.59(\mathrm{~s}, 3 \mathrm{H}$, $\left.\mathrm{CH}_{3}+\mathrm{OCH}_{3}\right), 3.89\left(\mathrm{~s}, 3 \mathrm{H}, \mathrm{CH}_{3}+\mathrm{OCH}_{3}\right), 4.07$ (s, 3H, $\left.\mathrm{CH}_{3}+\mathrm{OCH}_{3}\right), 4.07$ (d, $\left.J=9.2,3.4 \mathrm{~Hz}, 1 \mathrm{H}, 17-\mathrm{H}\right), 4.44$ (q, $J=7.1 \mathrm{~Hz}, 1 \mathrm{H}, 18-\mathrm{H}), 6.16$ (d, $\left.J=11.5 \mathrm{~Hz}, 1 \mathrm{H}, c_{s}-3 \mathrm{~b}-\mathrm{H}\right)$, $6.36(\mathrm{~d}, J=17.8 \mathrm{~Hz}, 1 \mathrm{H}$, trans $-3 \mathrm{~b}-\mathrm{H}), 6.10(\mathrm{~s}, 1 \mathrm{H}, 15 \mathrm{a}-$ $\mathrm{C}=\mathrm{CH}), 7.3(\mathrm{~s}, 1 \mathrm{H}, 15 \mathrm{a}-\mathrm{C}=\mathrm{CH}), 8.09(\mathrm{dd}, J=17.8,11.5$ $\mathrm{Hz}, 3 \mathrm{a}-\mathrm{H}), 8.83$ (s, 1H, meso-H), 9.67 (s, 1H, meso-H), $9.74(\mathrm{~s}, 1 \mathrm{H}$, meso-H); IR (KBr) v: $3458(\mathrm{~N}-\mathrm{H}), 2929$ $(\mathrm{C}-\mathrm{H}), 1743 \sim 1695(\mathrm{C}=\mathrm{O}), 1623(\mathrm{C}=\mathrm{C}), 1504$ (chlorin skeleton), 1467, 1384, 1226, 1031, 1018, $912 \mathrm{~cm}^{-1}$. Anal. calcd for $\mathrm{C}_{38} \mathrm{H}_{42} \mathrm{~N}_{4} \mathrm{O}_{6}$ : C 70.13, $\mathrm{H} \mathrm{6.51,} \mathrm{N} \mathrm{8.61;} \mathrm{found} \mathrm{C}$ 69.97, Н 6.48, N 8.79.

\section{$1.615^{1}$-红紫素-7 内酯二甲酯(10)的合成}

将 $120 \mathrm{mg}(0.184 \mathrm{mmol})$ 二氢卟吩 $-\mathrm{p}_{6} 2$ 溶解于 $8 \mathrm{~mL}$ 
二氯甲烷和 $8 \mathrm{~mL}$ 甲醇的混合液中, 于 $0{ }^{\circ} \mathrm{C}$ 搅拌下将解 于 $5 \mathrm{~mL}$ 甲醇中的 $40 \mathrm{mg}$ 硼氢化钠溶逐滴加入, TLC 监测 反应进程, 大约 $30 \mathrm{~min}$ 反应结束. 向反应体系中加入 25 $\mathrm{mL}$ 水和 $20 \mathrm{~mL}$ 二氯甲烷, 分出有机层, 无水硫酸钠干 燥后浓缩, 硅胶柱层析分离[展开剂: $V$ (石油醚) $: V($ 乙 酸乙酯 $)=3: 1]$, 分离出 $24 \mathrm{mg}(0.039 \mathrm{mmol})$ 红色固体 10, 产率 21\%. m.p. 217 $220{ }^{\circ} \mathrm{C}$; UV-vis $\left(\mathrm{CHCl}_{3}\right) \lambda_{\max }$

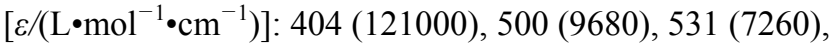
628 (21780), $668(41400) \mathrm{nm} ;{ }^{1} \mathrm{H}$ NMR $\left(\mathrm{CDCl}_{3}\right) \delta:-1.37$ $(-1.66)($ br s, $1 \mathrm{H}, \mathrm{NH}),-0.80(-1.13)(\mathrm{br} \mathrm{s}, 1 \mathrm{H}, \mathrm{NH})$, $1.70\left(\mathrm{t}, J=7.6 \mathrm{~Hz}, 3 \mathrm{H}, 8-\mathrm{CH}_{3}\right), 1.68(1.66)(\mathrm{d}, J=7.3 \mathrm{~Hz}$, $\left.3 \mathrm{H}, 18-\mathrm{CH}_{3}\right), 1.73(1.72)\left(\mathrm{d}, J=7.3 \mathrm{~Hz}, 3 \mathrm{H}, 18-\mathrm{CH}_{3}\right)$, $1.99 \sim 2.18(\mathrm{~m}, 1 \mathrm{H}, 17 \mathrm{a}+17 \mathrm{~b}-\mathrm{H}), 2.37 \sim 2.56(\mathrm{~m}, 1 \mathrm{H}$, $17 \mathrm{a}+17 \mathrm{~b}-\mathrm{H}), \quad 2.58 \sim 2.79(\mathrm{~m}, 2 \mathrm{H}, 17 \mathrm{a}+17 \mathrm{~b}-\mathrm{H}), 3.68$ (3.66) (q, $J=7.6 \mathrm{~Hz}, 1 \mathrm{H}, 8 \mathrm{a}-\mathrm{H}), 3.21$ (3.19) (s, $3 \mathrm{H}, \mathrm{CH}_{3}+$ $\mathrm{OCH}_{3}$ ), 3.42 (3.38) (s, 3H, $\mathrm{CH}_{3}+\mathrm{OCH}_{3}$ ), 3.74 (3.65) (s, $3 \mathrm{H}, \mathrm{CH}_{3}+\mathrm{OCH}_{3}$ ), 3.80 (3.87) (s, $3 \mathrm{H}, \mathrm{CH}_{3}+\mathrm{OCH}_{3}$ ), 4.05 (4.00) (s, 3H, $\left.\mathrm{CH}_{3}+\mathrm{OCH}_{3}\right), 4.49$ (4.47) (q, $J=7.6, \mathrm{~Hz}$, $1 \mathrm{H}, 18-\mathrm{H}), 4.54(4.46)(\mathrm{d}, J=8.0 \mathrm{~Hz}, 1 \mathrm{H}, 17-\mathrm{H}), 6.14$ (6.11) (dd, $J=11.6,1.1 \mathrm{~Hz}, 1 \mathrm{H}$, cis-3b-H), 6.28 (6.26) (d, $J=17.8,1.1 \mathrm{~Hz}, 1 \mathrm{H}$, trans-3b-H), $7.72(7.48)(\mathrm{s}, 1 \mathrm{H}$, $15 \mathrm{a}-\mathrm{H}), 7.98$ (7.94) (dd, $J=17.8,11.6 \mathrm{~Hz}, 1 \mathrm{H}, 3 \mathrm{a}-\mathrm{H}), 8.69$ (8.60) (s, 1H, meso-H), 9.49 (9.40) (s, 1H, meso-H), 9.67 (9.60) (s, 1H, meso-H); IR (KBr) v: 3396, $3317(\mathrm{~N}-\mathrm{H})$, 2958, $2927(\mathrm{C}-\mathrm{H}), 1726 \sim 1697(\mathrm{C}=\mathrm{O}), 1643(\mathrm{C}=\mathrm{C})$, 1542 (chlorin skeleton), 1461, 1269, 1068, 973, $732 \mathrm{~cm}^{-1}$. Anal. calcd for $\mathrm{C}_{36} \mathrm{H}_{38} \mathrm{~N}_{4} \mathrm{O}_{6}$ : C 69.44, H 6.15, N 9.00; found $\mathrm{C}$ 69.62, H 6.03, N 9.23.

\subsection{3-(1-羟基乙基)-红紫素-7 内酯三甲酯(11)的合成}

将 $96 \mathrm{mg}(0.147 \mathrm{mmol})$ 红紫素-7 三甲酯 2 溶于 10 $\mathrm{mL}$ 的含有 $30 \% \mathrm{HBr}$ 的乙酸溶液中, $50{ }^{\circ} \mathrm{C}$ 条件下恒温摚 拌. TLC 检测反应时间, 反应大约需 $4 \mathrm{~h}$. 向反应体系加 入 $30 \mathrm{~mL}$ 水, 然后用二氯甲烷萃取 $(50 \mathrm{~mL} \times 3)$, 合并有 机层, 然后用碳酸氢钠饱和溶液洗至溶液成中性, 无水 硫酸钠干燥后以重氮甲烷甲基化, 浓缩, 硅胶柱层析分 离[展开剂: $V$ (石油醚) $: V$ (乙酸乙酯 $)=2: 1$ ], 分离出 53 mg (0.079 mmol)绿色固体 11, 产率 54\%. m.p. 266 269 ${ }^{\circ} \mathrm{C}$; UV-vis $\left(\mathrm{CHCl}_{3}\right) \lambda_{\max }\left[\varepsilon /\left(\mathrm{L} \cdot \mathrm{mol}^{-1} \cdot \mathrm{cm}^{-1}\right)\right]: 414$ (120000), 519 (9600), 551 (15600), 622 (600), 681 (44400) nm; ${ }^{1} \mathrm{H}$ NMR $\left(\mathrm{CDCl}_{3}\right) \delta:-0.16$ (br s, $\left.1 \mathrm{H}, \mathrm{NH}\right)$, 0.07 (br s, $1 \mathrm{H}, \mathrm{NH}$ ), 1.66 (t, $\left.J=7.6 \mathrm{~Hz}, 8 \mathrm{~b}-\mathrm{CH}_{3}\right), 1.79$ (1.77) $\left(\mathrm{d}, J=7.2 \mathrm{~Hz}, 3 \mathrm{H}, 18-\mathrm{CH}_{3}\right), 2.04 \sim 2.14(\mathrm{~m}, 1 \mathrm{H}$, $17 \mathrm{a}+17 \mathrm{~b}-\mathrm{H}), 2.32 \sim 2.42(\mathrm{~m}, 2 \mathrm{H}, 17 \mathrm{a}+17 \mathrm{~b}-\mathrm{H}), 2.50 \sim$ $2.59(\mathrm{~m}, 1 \mathrm{H}, 17 \mathrm{a}+17 \mathrm{~b}-\mathrm{H}), 2.19(\mathrm{~d}, J=6.6 \mathrm{~Hz}, 3 \mathrm{H}$, $\left.3 \mathrm{~b}-\mathrm{CH}_{3}\right), 3.18$ (s, 3H, $\mathrm{CH}_{3}+\mathrm{OCH}_{3}$ ), 3.33 (3.32) (s, 3H,
$\left.\mathrm{CH}_{3}+\mathrm{OCH}_{3}\right), 3.53$ (3.52) (s, $\left.3 \mathrm{H}, \mathrm{CH}_{3}+\mathrm{OCH}_{3}\right), 3.60$ (s, $\left.3 \mathrm{H}, \mathrm{CH}_{3}+\mathrm{OCH}_{3}\right), 3.88\left(\mathrm{~s}, 3 \mathrm{H}, \mathrm{CH}_{3}+\mathrm{OCH}_{3}\right), 4.14(\mathrm{~s}, 3 \mathrm{H}$, $\left.\mathrm{CH}_{3}+\mathrm{OCH}_{3}\right), 3.62(\mathrm{q}, J=7.6 \mathrm{~Hz}, 2 \mathrm{H}, 8 \mathrm{a}-\mathrm{H}), 4.31(\mathrm{q}, J=$ $7.3 \mathrm{~Hz}, 1 \mathrm{H}, 18-\mathrm{H}), 4.67$ (d, $J=8.64 \mathrm{~Hz}, 1 \mathrm{H}, 17-\mathrm{H}), 630$ (q, $J=6.6 \mathrm{~Hz}, 1 \mathrm{H}, 3 \mathrm{a}-\mathrm{H}), 8.45$ (s, 1H, meso-H), 9.59 (s, 1H, meso-H), 9.60 (s, 1H, meso-H); IR (KBr) v: 3469, 3369 $(\mathrm{N}-\mathrm{H}), 2964,2931(\mathrm{C}-\mathrm{H}), 1739 \sim 1693(\mathrm{C}=\mathrm{O}), 1633$ $(\mathrm{C}=\mathrm{C}), 1544$ (chlorin skeleton), 1402, 1309, 1120, 1033, $912 \mathrm{~cm}^{-1}$. Anal. calcd for $\mathrm{C}_{37} \mathrm{H}_{42} \mathrm{~N}_{4} \mathrm{O}_{8}: \mathrm{C} 66.25, \mathrm{H} 6.31, \mathrm{~N}$ 8.35; found C 66.23, H 6.42, N 8.58.

\subsection{2-甲酰基-12-去甲基二氢卟吩- $p_{6}$-三甲酯(12)和} 12-甲酰基-12-去甲基红紫卟啉(13)的合成

在 $35 \mathrm{~mL}$ 二氯甲烷中溶解 $380 \mathrm{mg}(0.608 \mathrm{mmol})$ 二氢 卟吩- $\mathrm{p}_{6}$ 三甲酯 $\mathbf{4}$, 然后加入 $\mathrm{LiOH}$ 甲醇水溶液 $(0.4 \mathrm{~g}$ $\mathrm{LiOH}+5 \mathrm{~mL}$ 水, 再加 $20 \mathrm{~mL} \mathrm{MeOH}$ ), 室温条件下开口 剧烈摚拌 $4 \mathrm{~h}$ 后, 用醋酸将反应液调至酸性, 先后加入 $20 \mathrm{~mL}$ 二氯甲烷和 $40 \mathrm{~mL}$ 水分层, 分出有机相后用二氯 甲烷萃取水相 $(150 \mathrm{~mL} \times 3)$, 合并有机相, 用冷水洗涤一 次，无水硫酸钠干燥，用重氮甲烷乙醚溶液处理，减压 蒸除溶剂, 硅胶柱层析分离[展开剂： $V$ (石油醚)：V(乙 酸乙酯 $)=4: 1$ ], 得到 $43 \mathrm{mg}(0.067 \mathrm{mmol})$ 绿色固体 12 和 $11 \mathrm{mg}(0.018 \mathrm{mmol})$ 红色固体 $\mathbf{1 3}$.

12: 产率 11\%. m.p. 234 $237{ }^{\circ} \mathrm{C}$; UV-vis $\left(\mathrm{CHCl}_{3}\right)$

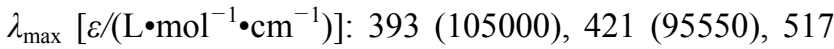
(13650), 644 (6300), 679 (66150) nm; ${ }^{1} \mathrm{H}$ NMR $\left(\mathrm{CDCl}_{3}\right)$ $\delta$ : $-0.81($ br s, $1 \mathrm{H}, \mathrm{NH}),-0.04($ br s, $1 \mathrm{H}, \mathrm{NH}), 1.66(\mathrm{t}$, $\left.J=7.6 \mathrm{~Hz}, 3 \mathrm{H}, 8-\mathrm{CH}_{3}\right), 1.80\left(\mathrm{~d}, J=7.2 \mathrm{~Hz}, 3 \mathrm{H}, 18-\mathrm{CH}_{3}\right)$, $2.01 \sim 2.06(\mathrm{~m}, 1 \mathrm{H}, 17 \mathrm{a}+17 \mathrm{~b}-\mathrm{H}), 2.07 \sim 2.16(\mathrm{~m}, 1 \mathrm{H}$, $17 \mathrm{a}+17 \mathrm{~b}-\mathrm{H}), 2.25 \sim 2.45(\mathrm{~m}, 2 \mathrm{H}, 17 \mathrm{a}+17 \mathrm{~b}-\mathrm{H}), 3.62(\mathrm{q}$, $J=7.6 \mathrm{~Hz}, 2 \mathrm{H}, 8 \mathrm{a}-\mathrm{H}), 3.06\left(\mathrm{~s}, 3 \mathrm{H}, \mathrm{CH}_{3}+\mathrm{OCH}_{3}\right), 3.24(\mathrm{~s}$, $3 \mathrm{H}, \mathrm{CH}_{3}+\mathrm{OCH}_{3}$ ), 3.55 (s, $\left.3 \mathrm{H}, \mathrm{CH}_{3}+\mathrm{OCH}_{3}\right), 4.13(\mathrm{~s}, 3 \mathrm{H}$, $\mathrm{CH}_{3}+\mathrm{OCH}_{3}$ ), 4.23 (s, $3 \mathrm{H}, \mathrm{CH}_{3}+\mathrm{OCH}_{3}$ ), 4.19 (q, $J=7.5$ $\mathrm{Hz}, 1 \mathrm{H}, 18-\mathrm{H}), 4.96$ (d, $J=8.7 \mathrm{~Hz}, 1 \mathrm{H}, 17-\mathrm{H}), 6.13$ (d, $J=$ $11.6 \mathrm{~Hz}, 1 \mathrm{H}$, trans-3b-H), $6.24(\mathrm{~d}, J=17.6 \mathrm{~Hz}, 1 \mathrm{H}$, cis-3b-H), 7.77 (dd, $J=17.6,11.6 \mathrm{~Hz}, 1 \mathrm{H}, 3 \mathrm{a}-\mathrm{H}), 8.27$ (s, $1 \mathrm{H}$, meso-H), 8.93 (s, 1H, meso-H), 10.39 (s, 1H, meso-H), 11.28 (s, 1H, CHO); IR (KBr) v: 3458, $3375(\mathrm{~N}-\mathrm{H})$, 2960, $2885(\mathrm{C}-\mathrm{H}), 1747 \sim 1703(\mathrm{C}=\mathrm{O}), 1635(\mathrm{C}=\mathrm{C})$, 1529 (chlorin skeleton), 1406, 1311, 1081, $910 \mathrm{~cm}^{-1}$. Anal. calcd for $\mathrm{C}_{36} \mathrm{H}_{38} \mathrm{~N}_{4} \mathrm{O}_{7}$ : C 67.70, H 6.00, N 8.77; found $\mathrm{C} 67.53, \mathrm{H} 5.91, \mathrm{~N} 8.86$.

13: 产率 3\%. m.p. 209 $211{ }^{\circ} \mathrm{C}$; UV-vis $\left(\mathrm{CHCl}_{3}\right)$ $\lambda_{\max }\left[\varepsilon /\left({\left.\left.\mathrm{L} \cdot \mathrm{mol}^{-1} \cdot \mathrm{cm}^{-1}\right)\right]:} 393\right.\right.$ (110000), 421 (100100), 517 (14300), 644 (6600), 679 (69300) nm; ${ }^{1} \mathrm{H}$ NMR $\left(\mathrm{CDCl}_{3}\right) \delta$ : -3.86 (br s, $2 \mathrm{H}, \mathrm{NH}), 1.86$ (t, $J=7.6 \mathrm{~Hz}, 3 \mathrm{H}, 8-\mathrm{CH}_{3}$ ), 
2.26 (q, $J=7.1,2 \mathrm{H}, 17 \mathrm{~b}-\mathrm{H}), 3.32(\mathrm{t}, J=7.8 \mathrm{~Hz}, 2 \mathrm{H}$, $17 \mathrm{a}-\mathrm{H}), 3.62\left(\mathrm{~s}, 3 \mathrm{H}, \mathrm{CH}_{3}+\mathrm{OCH}_{3}\right), 3.65\left(\mathrm{~s}, 3 \mathrm{H}, \mathrm{CH}_{3}+\right.$ $\left.\mathrm{OCH}_{3}\right), 3.66\left(\mathrm{~s}, 3 \mathrm{H}, \mathrm{CH}_{3}+\mathrm{OCH}_{3}\right), 3.95\left(\mathrm{~s}, 3 \mathrm{H}, \mathrm{CH}_{3}+\right.$ $\mathrm{OCH}_{3}$ ), 4.11 (q, $\left.J=7.6 \mathrm{~Hz}, 2 \mathrm{H}, 8 \mathrm{a}-\mathrm{H}\right), 6.14$ (dd, $J=11.4$, $1.5 \mathrm{~Hz}, 1 \mathrm{H}$, cis-3b-H), 6.39 (dd, $J=17.8,1.5 \mathrm{~Hz}, 1 \mathrm{H}$, trans-3b-H), 8.21 (dd, $J=17.8,11.4 \mathrm{~Hz}, 1 \mathrm{H}, 3 \mathrm{a}-\mathrm{H}), 9.98$ $(\mathrm{s}, 1 \mathrm{H}$, meso-H), $10.04(\mathrm{~s}, 1 \mathrm{H}$, meso-H), $10.11(\mathrm{~s}, 1 \mathrm{H}$, meso-H), 11.06 (s, 1H, 12-CHO); IR (KBr) v: $3446(\mathrm{~N}-$ H), $2931(\mathrm{C}-\mathrm{H}), 1743 \sim 1704(\mathrm{C}=\mathrm{O}), 1633(\mathrm{C}=\mathrm{C})$, 1558 (chlorin skeleton), 1475, 1384, 1228, 1168, 1078, $948 \mathrm{~cm}^{-1}$. Anal. calcd for $\mathrm{C}_{34} \mathrm{H}_{30} \mathrm{~N}_{4} \mathrm{O}_{6}: \mathrm{C} 69.14, \mathrm{H} 5.12, \mathrm{~N}$ 9.49; found C 69.29, H 5.32, N 9.73.

\subsection{2-羟乙基-12-去甲基卟吩- $\mathrm{p}_{6}$ 三甲酯(14)的合成}

将 $212 \mathrm{mg}(0.339 \mathrm{mmol})$ 二氢卟吩 $-\mathrm{p}_{6}$ 三甲酯 4 溶于 $10 \mathrm{~mL}$ 冰乙酸中, 然后搅拌下先后加入 $8 \mathrm{~mL}$ 浓磷酸、 15 $\mathrm{mL}$ 浓盐酸和 $20 \mathrm{~mL} 40 \%$ 的甲醛水溶液. 室温摚拌 $3 \mathrm{~d}$ 后, 加入 $25 \mathrm{~mL}$ 水, 用二氯甲烷萃取 $(50 \mathrm{~mL} \times 3)$, 合并有机 相, 水洗涤除酸, 经无水硫酸钠干燥后用重氮甲烷甲基 化, 减压蒸除溶剂, 硅胶柱层析分离[展开剂: $V($ 石油 醚) $: V$ (乙酸乙酯 $)=4: 1]$, 得到 $40 \mathrm{mg}(0.061 \mathrm{mmol})$ 绿 色固体 14, 产率 $18 \%$. m.p. $246 \sim 249{ }^{\circ} \mathrm{C}$; UV-vis

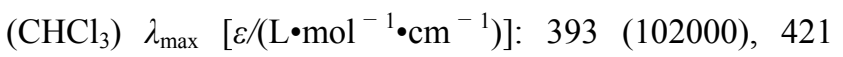
(92820), 517 (13260), 644 (6120), 656 (64260) nm; ${ }^{1} \mathrm{H}$ $\operatorname{NMR}\left(\mathrm{CDCl}_{3}\right) \delta:-0.81($ br s, $1 \mathrm{H}, \mathrm{NH}),-0.04(\mathrm{br} \mathrm{s}, 1 \mathrm{H}$, $\mathrm{NH}), 1.66\left(\mathrm{t}, J=7.6 \mathrm{~Hz}, 3 \mathrm{H}, 8-\mathrm{CH}_{3}\right), 1.80(\mathrm{~d}, J=7.2 \mathrm{~Hz}$, $\left.3 \mathrm{H}, 18-\mathrm{CH}_{3}\right), 2.01 \sim 2.06(\mathrm{~m}, 1 \mathrm{H}, 17 \mathrm{a}+17 \mathrm{~b}-\mathrm{H}), 2.07 \sim$ $2.16(\mathrm{~m}, 1 \mathrm{H}, 17 \mathrm{a}+17 \mathrm{~b}-\mathrm{H}), 2.25 \sim 2.45(\mathrm{~m}, 2 \mathrm{H}, 17 \mathrm{a}+$ 17b-H), 3.62 (q, $J=7.6 \mathrm{~Hz}, 2 \mathrm{H}, 8 \mathrm{a}-\mathrm{H}), 3.06$ (s, 3H, $\mathrm{CH}_{3}+$ $\left.\mathrm{OCH}_{3}\right), 3.24\left(\mathrm{~s}, 3 \mathrm{H}, \mathrm{CH}_{3}+\mathrm{OCH}_{3}\right), 3.55\left(\mathrm{~s}, 3 \mathrm{H}, \mathrm{CH}_{3}+\right.$ $\left.\mathrm{OCH}_{3}\right), 4.13$ (s, 3H, $\left.\mathrm{CH}_{3}+\mathrm{OCH}_{3}\right), 4.23\left(\mathrm{~s}, 3 \mathrm{H}, \mathrm{CH}_{3}+\right.$ $\left.\mathrm{OCH}_{3}\right), 4.19$ (q, $\left.J=7.5 \mathrm{~Hz}, 1 \mathrm{H}, 18-\mathrm{H}\right), 4.96(\mathrm{~d}, J=8.7 \mathrm{~Hz}$, $1 \mathrm{H}, 17-\mathrm{H}), 6.13(\mathrm{~d}, J=11.6 \mathrm{~Hz}, 1 \mathrm{H}$, trans-3b-H), $6.24(\mathrm{~d}$, $J=17.6 \mathrm{~Hz}, 1 \mathrm{H}, c i s-3 \mathrm{~b}-\mathrm{H}), 7.77$ (dd, $J=17.6,11.6 \mathrm{~Hz}$, 1H, 3a-H), 8.27 (s, 1H, meso-H), 8.93 (s, 1H, meso-H), 10.39 (s, 1H, meso-H), 11.28 (s, 1H, CHO); IR (KBr) v: $3446(\mathrm{~N}-\mathrm{H}), 2927,2860(\mathrm{C}-\mathrm{H}), 1743 \sim 1726(\mathrm{C}=\mathrm{O})$, $1633(\mathrm{C}=\mathrm{C}), 1558$ (chlorin skeleton), 1463, 1396, 1099, $800 \mathrm{~cm}^{-1}$. Anal. calcd for $\mathrm{C}_{37} \mathrm{H}_{42} \mathrm{~N}_{4} \mathrm{O}_{7}: \mathrm{C} 67.87, \mathrm{H} 6.47, \mathrm{~N}$ 8.56; found C 67.59, H 6.42, N 8.44.

\section{2 结果与讨论}

\section{1 多酯基二氢卟吩的 C-D 环端向酯基与化学反应活} 性

由于二氢卟吩- $\mathrm{p}_{6}$ 或者红紫素-7 的 13-和 15-位上酯 基可以绕着连接于母环的化学键自由旋转, 因而这些取
代基团中的碳氧双键与大环的共轭程度相对较低, 对大 环分子的化学性质所施加的影响也不尽相同. 以 15 -位 酯基为例：在构型 $\mathbf{I}$ 中的 C(15)-碳氧双键与大环平面平 行，此时与氮杂轮烯芳香体系形成较好的共轭体系; 如 果沿着 $\mathrm{C}(15)-\mathrm{C}(15 \mathrm{a})$ 轴旋转 $90^{\circ}$ 后, 所形成的构象 II 中 的碳氧双键则与母环平面相互垂直, 二者基本不会发生 任何 $\pi$-电子离域(图 1). 与红紫素系列衍生物的刚性外 接环羰基相比，尽管在 C-D 环端向同样连有两个羰基, 但二氢卟吩- $\mathrm{p}_{6}$ 三甲酯 2 和红紫素-7 三甲酯 4 的最大可 见光吸收分别为 669 和 $676 \mathrm{~nm}$, 而相应的红紫素-18 甲 酯 3 的相应波长则达到 $700 \mathrm{~nm}^{[12]}$, 较短的 $Q_{\mathrm{y}}$ 吸收波长 也间接地说明, 沿着 $\mathrm{N}^{21}-\mathrm{N}^{23}$ 轴向的外接直链酯基不能 象环酕那样与大环色基建立良好的共轭关系.

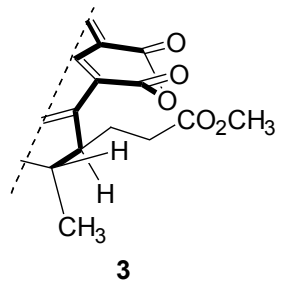

羰基始终与色基共平面

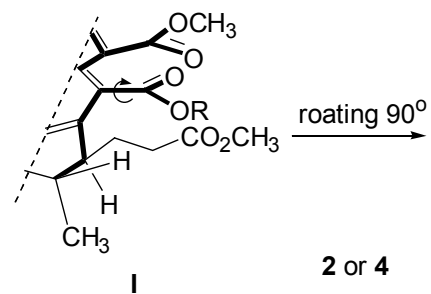

15-羰基与色基共平面

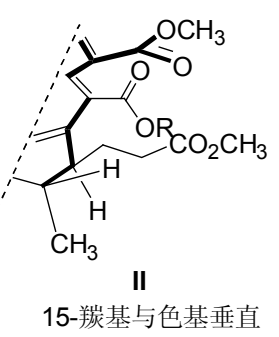

15-羰基与色基垂直
图 1 二氢卟吩 2 4 的 13-和 15-位取代基与大环色基的共轭 结构

Figure 1 The conjugation structures of the substitutes at 13and 15-position of chlorins $2 \sim 4$

环上所连接的吸电子 $\mathrm{C}=\mathrm{O}$ 双键的电子效应降低了 环上的电流密度, 由此而来对二氢卟吩的化学反应也将 产生非常明显的影响. 外接羰基与大环色基的共轭程度 越低, 环电流的电流密度降低幅度越小, 更有可能在 20-位上发生亲电取代反应. 与红紫素-8 甲酯 3 相比, 红 紫素-7 三甲酯 2 的硝化反应则相对容易，而前者甚至不 能够发生澳代反应 ${ }^{[12]}$. 相反, 这些吸电子性羰基离散 $\mathrm{C}$ (3)-乙烯基的 $\pi$-键电子云的电子效应, 又可以提高其 1,3 -偶极环加成的反应速度. 本文中二氢卟吩- $\mathrm{p}_{6}$ 三甲酯 4 与重氮甲烷的环加成反应需要 $32 \mathrm{~h}$, 而在相同的条件 下, 红紫素-18 3 只需一半的时间就可以完成相应的化 学反应 ${ }^{[13]}$.

相对脱镁叶绿素酸-a 1 而言, 只有一个吸电子羰基 直接与大环色基相连, 其环电流的密度显然要高于红紫 
素-7 2 和二氢卟吩- $\mathrm{p}_{6}$ 4. 由于后者的两个 $\mathrm{C}=\mathrm{O}$ 双键共 同对大环的芳香体系施加吸电子效应，因此在更深程度 上钝化了 20-meso-位的亲电反应活性. 在酸性环境中, 脱镁叶绿酸-a 甲酯可以与 40\%的甲醛在 20-meso-位上顺 利地完成了亲电性 Blanc 氯甲基化反应 ${ }^{[14]}$. 化合物 4 在 此条件下却没有形成相应的亲电取代历程, 而是在 12位的甲基上发生了类似于 Aldol 羟醛缩合反应.

\section{2 二氢卟吩- $\mathrm{p}_{6}$ 三甲酯的 $\mathrm{C}(12)$-甲基的化学反应}

与其他具有刚性外接环结构的叶绿素-a 衍生物一 样，二氢卟吩- $\mathrm{p}_{6}$ 三甲酯 4 的 12-位甲基同样具有一定的 酸性 ${ }^{[7]}$. 在空气氧化反应中, 氢氧化锂促进的 13-位酯羰 基的烯醇化给出烯醇式负氧离子 $\mathbf{A}$, 然后与空气中的氧 分子发生反应而转化成连有过氧氢结构的中间体 $\mathbf{B}$; 12位甲基在相同条件下再度失去质子完成第二次烯醇异 构, 所形成的中间体 $\mathbf{C}$ 经过电子转移生成二氢卟吩醛 12. 其 13-位酯基继续发生㿝化并与邻位酯基成酐, 进 而转化成 12-甲酰基红紫素-18 D. 由于该分子在 12-, 13-
和 15-位上均连有羰基，在很大程度上提升了 17-位氢原 子的酸性. 在氢氧负离子的作用下, 经过跨环电子转移 后其外接环酤形成了烯醇异构体 $\mathbf{E}$ ，随之与空气氧分子 发生反应并给出红紫素-18 过氧化氢中间体 $\mathbf{F}$, 最后, 脱 去一分子过氧化氢生成红紫卟啉 13. 在酸性条件下, 化 合物 4 的 13-位酯羰基质子化形成中间体 $\mathbf{G}$, 在碱质点 (各种酸根或甲醛)的促进下完成其异构化, 烯醇式异构 体 $\mathbf{H}$ 再与甲醛进行亲核加成反应，从反应体系中捕获质 子后得到 12-羟乙基取代的二氢卟吩酯 14(图 2).

多酯基取代的叶绿素衍生物显示出与脱镁叶绿酸 相似的化学活性, 所合成的新型二氢卟吩的最大可见光 的吸收波长均处于光动力疗法的治疗窗内, 其 C-D 环端 向的多酯基结构所具有的亲水性能，与 A-B 环端向的脂 溶性基团能够形成良好的两亲特性, 在一定程度上改善 了四吡咯大环分子的生物利用度, 进而可以大幅度提高 光敏剂的光动力抗癌功效.<smiles>C=C1N=C(/C(C(=O)OC)=c2\[nH]c(=C)c(C)c2C(=O)O)C(CCC(=O)OC)[C@H]1C</smiles>

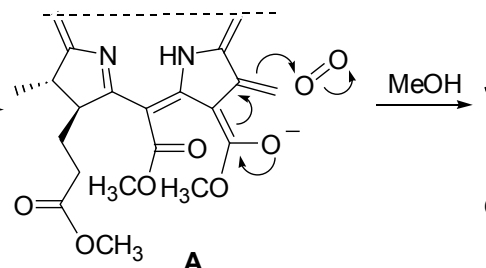<smiles>C=c1[nH]/c(=C(\C(=O)OC)C2=NC(=O)[C@@H](C)[C@@H]2CCC(=O)OC)c(C(=O)OC)c1C(=O)OO</smiles>

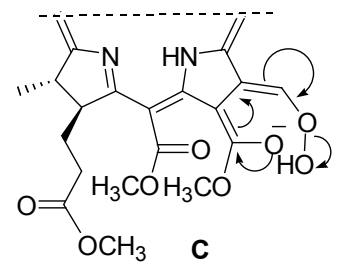<smiles>[B]CCc1c(C(=O)OC)c(=C(C(=O)OC)C2=NC(=C)[C@H](C)C2CC)[nH]c1=C</smiles>

G

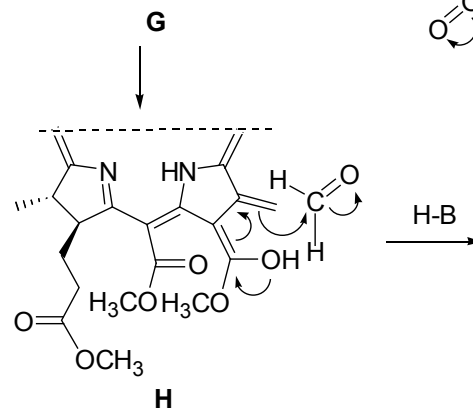

H

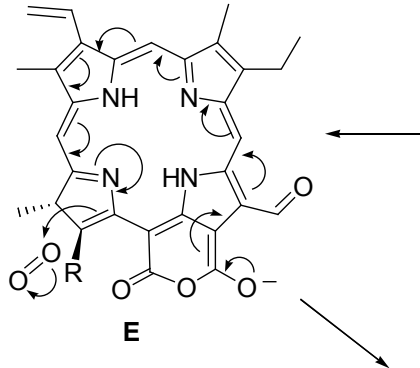

$\mathrm{H}-\mathrm{B}$

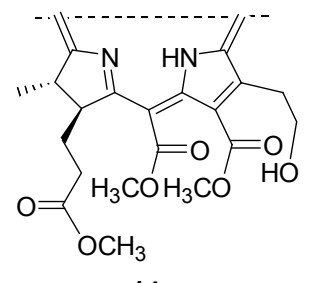

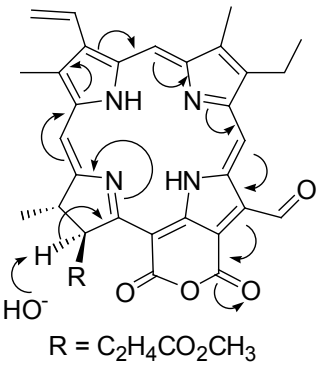

D

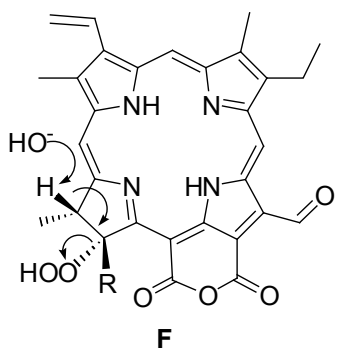

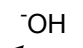<smiles>C=C1N=C(C(C(=O)OC)=c2[nH]c(=C)c(C=O)c2C(=O)OC)C(CCC(=O)OC)[C@@H]1C</smiles>

12

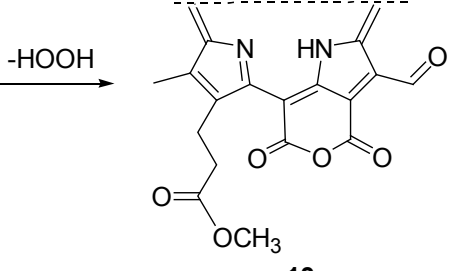

13

图 2 二氢卟吩- $\mathrm{p}_{6}$ 三甲酯 4 的空气氧化和 Aldol 反应机理

Figure 1 The allomerization and aldol reaction of chlorine- $\mathrm{p}_{6}$ trimethyl ester 4

\section{References}

[1] Pavlov, V. Y.; Ponomarev, G. V. Chem. Heterocycl. Compd. 2004, 40, 393.

[2] Grin, M. A.; Titeev, R. A.; Brittal, D. I.; Ulybina, O. V.;
Tsiprovskiy, A. G.; Berzina, M. Y.; Lobanova, I. A.; Sivaev, I. B.; Bregadzeb, V. I.; Mironova, A. F. Mendeleev Commun. 2011, 21, 84.

[3] Wang, J.-J. Chin. J. Org. Chem. 2005, 25, 1353 (in Chinese). (王进军, 有机化学, 2005, 25, 1353.) 
[4] Haas, R. N. S.; Jong, R. L. P.; Noushazare, M.; Erkelens, K.; Smijs, T. G. M.; Liu, Y.; Gast, P.; Schuitmaker, H. J.; Lugtenhurg, J. Eur. J. Org. Chem. 2004, 4024.

[5] Kozyrev, A. N.; Chen, Y.-H.; Goswami, L. N.; Tabaczynski, W. A.; Pandey, R. K. J. Org. Chem. 2006, 71, 1949.

[6] Morishita, H.; Tamiaki, H. Bioorg. Med. Chem. 2003, 11, 4049.

[7] Li, J.-J.; Liu, W.-H.; Li, F.-G.; Wang, J.-J.; Suo, Y.-R.; Liu, Y.-J. Chin. J. Org. Chem. 2007, 27, 1594 (in Chinese). (李家柱, 刘万卉, 李付国, 王进军, 索有瑞, 刘永军, 有机化学, 2007, 27, 1594.)

[8] Wang, J.-J.; Li, J.-J.; Yin, J.-G.; Liu, Y.-J. Chin. J. Org. Chem. 2008, 28, 693 (in Chinese).

(王进军, 李家柱, 殷军港, 刘永军, 有机化学, 2008, 28, 693.)

[9] Wang, J.-J.; Zhang, P.; Wang, P.; Chen, G.-L.; Li, F. G. Chin. J. Org. Chem. 2010, 30, 1192 (in Chinese ).
(王进军, 张朋, 王朋, 陈冠龙, 李付国, 有机化学, 2010, 30, 1192.)

[10] Wang, J.-J.; Li, F.-G.; Li, Y.-W. Chin. J. Org. Chem. 2011, 31, 68 (in Chinese). (王进军, 李付国, 李韵伟, 有机化学, 2011, 31, 68.)

[11] Smith, K. M.; Gogg, D. A.; Simpson, D. J. J. Am. Chem. Soc. 1985 , 107,4946

[12] Wang, P. M.S. Thesis, Yantai University, Yantai, 2009 (in Chinese).

(王朋, 硕士论文, 烟台大学, 烟台, 2009.)

[13] Wang, J.-J.; Li, J.-Z.; Gryko, D.; Shim, Y.-K. Bull. Korea Chem. 2006, 27, 1083.

[14] Zhang, P. M.S. Thesis, Yantai University, Yantai, 2010 (in Chinese).

(张朋, 硕士论文, 烟台大学, 烟台, 2010.)

(Sun, H.; Lu, Z. 